\title{
Comparison of two preclinical myocardial infarct models: coronary coil deployment versus surgical ligation
}

\author{
Carolina Gálvez-Montón 1*, Cristina Prat-Vidal', Idoia Díaz-Güemes², Verónica Crisóstomo², Carolina Soler-Botija ${ }^{1}$, \\ Santiago Roura', Aida Llucià-Valldeperas ${ }^{1}$, Isaac Perea-Gil ${ }^{1}$, Francisco M Sánchez-Margallo² and Antoni Bayes-Genis ${ }^{1,3,4}$
}

\begin{abstract}
Background: Despite recent advances, myocardial infarction (MI) remains the leading cause of death worldwide. Pre-clinical animal models that closely mimic human Ml are pivotal for a quick translation of research and swine have similarities in anatomy and physiology. Here, we compared coronary surgical ligation versus coil embolization Ml models in swine.

Methods: Fifteen animals were randomly distributed to undergo surgical ligation $(n=7)$ or coil embolization ( $n=8)$. We evaluated infarct size, scar fibrosis, inflammation, myocardial vascularization, and cardiac function by magnetic resonance imaging (MRI).

Results: Thirty-five days after $M I$, there were no differences between the models in infarct size $(P=0.53)$, left ventricular $(\mathrm{LV}$ ) ejection fraction $(P=0.19)$, LV end systolic volume $(P=0.22)$, LV end diastolic volume $(P=0.84)$, and cardiac output $(P=0.89)$. Histologically, cardiac scars did not differ and the collagen content, collagen type I (I), collagen type III (III), and the I/III ratio were similar in both groups. Inflammation was assessed using specific anti-CD3 and anti-CD25 antibodies. There was similar activation of inflammation throughout the heart after coil embolization $(P=0.78)$; while, there were more activated lymphocytes in the infarcted myocardium in the surgical occlusion model $(P=0.02)$. Less myocardial vascularization in the infarction areas compared with the border and remote zones only in coil embolization animals was observed ( $P=0.004$ and $P=0.014$, respectively).

Conclusions: Our results support that surgical occlusion and coil embolization MI models generate similar infarct size, cardiac function impairment, and myocardial fibrosis; although, inflammation and myocardial vascularization levels were closer to those found in humans when coil embolization was performed.
\end{abstract}

Keywords: Myocardial infarction, Magnetic resonance imaging, Animal model surgery, Coil embolization, Surgical occlusion

\section{Background}

Myocardial infarction (MI) is the leading cause of morbidity, mortality, and disability in the worldwide and remains one of the greatest challenges in biomedical research [1]. Current treatments including primary percutaneous coronary intervention, coronary artery bypass graft, or thrombolysis have significantly improved

\footnotetext{
* Correspondence: cgalvezmonton@gmail.com

'ICREC (Heart Failure and Cardiac Regeneration) Research Program, IGTP, Cardiology Service, Hospital Universitari Germans Trias i Pujol, Crta. Can Ruti, Camí de les Escoles, s/n, 08916 Badalona, Barcelona, Spain

Full list of author information is available at the end of the article
}

outcomes but do not restore myocardial damage after MI [2]. Consequently, new strategies such as cardiac cell therapy, tissue engineering, and gene therapy are being evaluated in pre-clinical models of disease [3]. Thus, for clinical translation, it is necessary to identify the best experimental model that mimics human MI conditions.

Numerous animal models evaluate the pathophysiological mechanisms of ventricular remodeling and MI development and progression [4,5]. Briefly, swine are mostly used because of their high similarity to humans, as they share minimal pre-existing coronary collaterals and have a similar coronary physiology and anatomy [6].

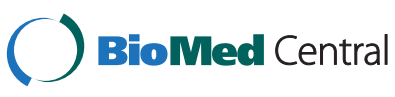

(c) 2014 Gálvez-Montón et al.; licensee BioMed Central Ltd. This is an Open Access article distributed under the terms of the Creative Commons Attribution License (http://creativecommons.org/licenses/by/2.0), which permits unrestricted use, distribution, and reproduction in any medium, provided the original work is properly credited. The Creative Commons Public Domain Dedication waiver (http://creativecommons.org/publicdomain/zero/1.0/) applies to the data made available in this article, unless otherwise stated. 
Despite acceleration of early myocardial healing process in swine compared to humans [7], histopathologic changes after MI are common with an earliest myocardial necrosis followed by infiltration of myofibroblast and inflammatory cells (i.e. neutrophils, macrophages, lymphocytes and plasma cells), and necrotic tissue replacement by collagen I and III [8]. MI pig model by permanent arterial occlusion can be induced following intracoronary ethanol administration [9], cryo-injury, cauterization [6], surgical ligation [10], and coil embolization [11]. Although ethanol, cryo-injury and cauterization are feasible and easily reproducible, these techniques promote pathophysiological changes different than those observed in humans [6]. Surgical coronary ligation causes irreversible damage to the myocardium and has been used extensively to assess cardiac regeneration following cell-based therapy and tissue engineering approaches. However, in contrast to human MI, the downside of this model involves an open-chest surgery. Alternatively, closed-chest options based on permanent coronary occlusion by coil deployment avoid the hassles of surgery, adjacent scarring, and post-operative inflammation.

The presented work aims to compare infarct size, histological traits and cardiac functional changes following surgical ligation or coil deployment. All interventions were performed in the first marginal branch of the circumflex coronary artery in swine and the follow-up period was 35 days.

\section{Methods}

\section{Study design}

Fifteen Landrace $\times$ Large White female pigs $(20-30 \mathrm{~kg}$ ) were randomly distributed into surgical coronary ligation $(\mathrm{n}=7)$ and coronary coil deployment $(\mathrm{n}=8)$ groups. All animals underwent $\mathrm{MI}$ induction in the first marginal branch of the circumflex coronary artery and were sacrificed after 35 days of follow-up. This study was approved by the local Animal Experimentation Unit Ethical Committee and complies with all guidelines concerning the use of animals in research and teaching as defined by the Guide for the Care and Use of Laboratory Animals (NIH Publication No. 80-23, revised 1996).

\section{Anaesthesia and analgesia}

Pigs were premedicated as previously described [7]. In brief, propofol (4 mg/kg, IV; Recofol ${ }^{\ominus}$, Bayer Schering Pharma) was used as an anaesthetic and, after endotracheal intubation, anaesthesia was maintained by $2 \%$ sevoflurane inhalation. A continued IV infusion of Ketorolac $\left(0.15 \mathrm{mg} / \mathrm{kg} / \mathrm{h}\right.$; Toradol $^{\circledR}$, Roche) and tramadol (0.5 mg/kg/h; Adolonta ${ }^{\oplus}$, Grünenthal Pharma, S.A.) was used as an intra-operatory analgesia. At the beginning of the surgical procedures, a $1 \mathrm{mg} / \mathrm{kg}$ lidocaine bolus (IV, Lidocaína $2 \%$, B. Braun) was administered, followed by a continuous infusion $(1 \mathrm{mg} / \mathrm{kg} / \mathrm{h})$ that was maintained for $1 \mathrm{~h}$ after MI induction. Finally, an IM dose of $2.5 \mathrm{mg} / \mathrm{kg}$ tulathromycin (Draxxin ${ }^{\circ}$, Pfizer Animal Health) was administered as a post-operative antibiotic. All surgery procedures were done under monitoring conditions with electrocardiogram (ECG) registration and measures of capnography, non-invasive arterial blood pressure, pulse oximetry, and temperature.

\section{Chronic MI models \\ Surgical coronary ligation}

MI was induced in 7 animals after coronary surgical ligation of the first marginal branch of the circumflex coronary artery (Figure 1A-B) as previously described [10]. Briefly, after lateral thoracotomy, MI was induced by a double-ligation (Prolene 5/0 W-8556 12-S, Ethicon Inc.) of the coronary artery, $1.5-\mathrm{cm}$ distal from the atrioventricular groove The onset of ST segment elevation on the ECG and bruising of the affected tissue were used to assess MI.

\section{Coil deployment}

Eight animals underwent a coronary coil embolization in the first marginal branch of the circumflex coronary artery (Figure 1C-D) as previously described [11]. Briefly, after engaging the left main artery using a 6-F AR1 guide catheter, a left lateral MI was experimentally induced by percutaneous embolization coil deployment (VortX-18 Diamond $3 \mathrm{~mm} / 3.3 \mathrm{~mm}$ coil, Boston Scientific/Target) in the first marginal branch of the circumflex artery under continuous fluoroscopy (BV Pulsera, Philips Medical Systems). In all cases, complete thrombotic occlusion (TIMI flow score $=0$ ) was confirmed by coronary angiography 15 min after coil implantation.

\section{Cardiac function assessment}

Cardiac function was evaluated by magnetic resonance imaging (MRI) performed at $1.5 \mathrm{~T}$ (Intera, Philips, Best, The Netherlands) using a phased-array cardiac coil $[10,11]$. All images were acquired under apnoea with continuous ECG gating. Left ventricular (LV) global function was assessed at baseline and at sacrifice, as previously described [6], and the ejection fraction (LVEF), end systolic volume (LVESV), end diastolic volume (LVEDV), and cardiac output (CO) were measured and compared between groups.

\section{Morphometric analysis}

After premedication, pigs were sacrificed with a potassium chloride IV injection. LV infarct size was blindly measured in digitally photographed transverse heart sections from $1 \mathrm{~cm}$ distally to the coronary ligation or coil localization. Additionally, in order to confirm a correlation of MRI findings with gross pathology findings, LV infarct 


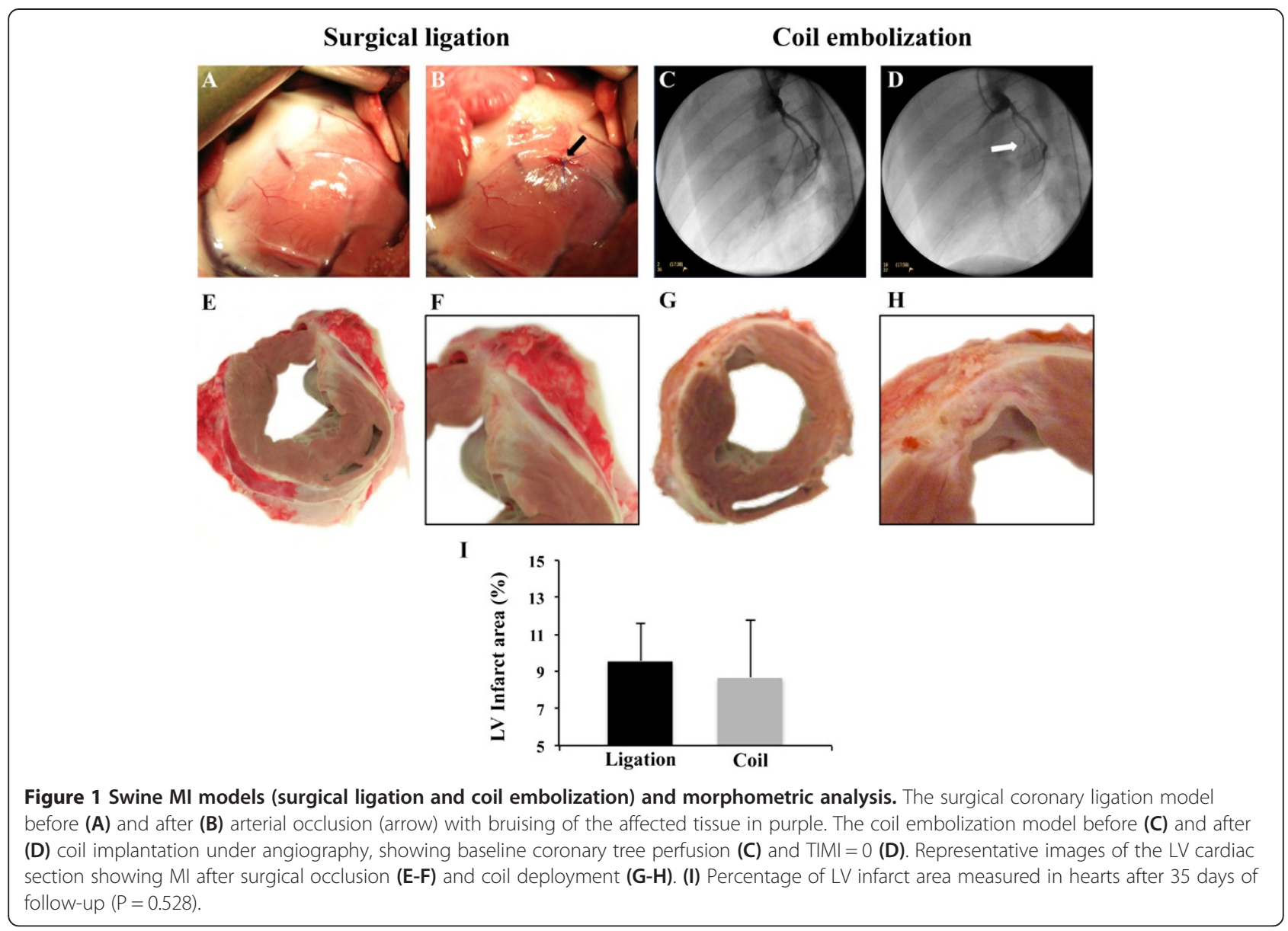

size was also analysed in gadolinium enhancement MRI images. Image-Pro Plus software (6.2.1 version; Media Cybernetics, Inc.) was used for quantitative morphometric measurements.

\section{Histological analysis}

After sacrifice, hearts were excised and samples from the infarct core and remote myocardium were snap frozen. After 10\% buffered formalin fixation, $5 \mu \mathrm{m}$ tissue sections were stained using Masson's and Gallego's trichrome procedures for histological examination. In addition, Picrosirius red staining was performed to analyse myocardial fibrosis, as previously described [6]. The collagen volume fraction (CVF), collagen I, collagen III, and collagen I/III ratio were measured in the infarct core and remote zones with ImagePro Plus software.

\section{Immunohistochemistry}

In MI sections, inflammation was assessed by measuring the total anti-CD3 antibody positive lymphocytes, those labelled with anti-CD25 antibody, and the CD25/CD3 ratio. Briefly, sections were incubated with primary antibodies against CD3 (1:100; AbD Serotec) and CD25 (1:10; AbD
Serotec) and with conjugated secondary antibodies using donkey anti-rat DyLight 488 (1:1000; Jackson ImmunoResearch) and donkey anti-mouse Cy3 (1:1000; Jackson ImmunoResearch), respectively. Vessel density in infarct, border, and remote zones was double-blindly assessed by Isolectin-B4 staining (1:25; Vectors Labs), Alexa Fluor 488conjugated Streptavidin (1:500; Vectors Labs). Images were captured with a Leica TCS SP5 microscope (20x) and results were expressed as a percentage of the vessel area per field [8]. All sections were finally counterstained with Hoechst 33342 (1:10000; Sigma-Aldrich) and analysed with Image-Pro Plus software.

\section{Statistical analysis}

Data are presented as the mean \pm SD. All analyses were performed with SPSS statistic software (19.0.1 version, SPSS Inc.). Differences in morphometric and histological data between the surgical ligation and coil deployment groups were analysed using Student's t-test and cardiac function evolution was compared using ANOVA with the Greenhouse-Geisser correction. Statistical significance was achieved when $\mathrm{P}<0.05$. 


\section{Results}

None of the animals died during MI induction or follow-up and were sacrificed at the pre-defined time course $(36 \pm 1.5$ surgical ligation vs. $37 \pm 1.5$ days coil embolization, respectively; $\mathrm{P}=0.28$ ).

\section{Infarct size}

Morphometric analysis from digitally photographed transverse heart sections revealed that LV infarct sizes (Figure 1E-H) were similar when comparing surgical ligation and coil embolization groups at sacrifice $(9.6 \pm 2$ vs. $8.7 \pm 3.1 \%$, respectively; $\mathrm{P}=0.53$ ) (Figure $1 \mathrm{I}$ ). This result was also confirmed when LV infarct size was calculated in gadolinium enhancement MRI images $(9.1 \pm 6.6$ vs. $11.6 \pm 4.6 \%$, respectively; $\mathrm{P}=0.26$ ).

\section{Cardiac function}

Baseline cardiac function was similar in both groups; in the surgical ligation versus coil embolization group with regards to LVEF ( $48 \pm 6$ vs. $47 \pm 8 \%$, respectively; $\mathrm{P}=$ $0.95)$, LVESV ( $25 \pm 5$ vs. $26 \pm 4 \mathrm{~mL}$, respectively; $\mathrm{P}=$ $0.62)$, and LVEDV ( $55 \pm 10$ vs. $49 \pm 5 \mathrm{~mL}$, respectively; $\mathrm{P}=0.16$ ). At sacrifice, ventricular function measurements were: LVEF ( $51 \pm 6$ vs. $56 \pm 9 \%$; $\mathrm{P}=0.19$ ), LVESV ( $32 \pm 3$ vs. $29 \pm 7 \mathrm{~mL} ; \mathrm{P}=0.22$ ), and LVEDV ( $66 \pm 5$ vs. $65 \pm 9 \mathrm{~mL} ; \mathrm{P}=0.84$ ). Although the measured baseline $\mathrm{CO}$ values were different between surgical and coil occlusion animals $(2.6 \pm 0.7$ vs. $1.9 \pm 0.3 \mathrm{~L} / \mathrm{min}$, respectively; $\mathrm{P}=0.02)$, they did not differ at sacrifice $(3.3 \pm 0.6$ vs. $3.3 \pm$ $0.8 \mathrm{~L} / \mathrm{min}$, respectively; $\mathrm{P}=0.89)$ (Online Table 1 ).

To ensure a correct analysis of cardiac function, Greenhouse-Geisser analysis was applied to take into account the inter-individual differences in its evolution in each animal (baseline vs. sacrifice) when the two groups were compared. The results confirmed that there were no differences between groups in LVEF $(\mathrm{P}=0.34)$, $\operatorname{LVESV}(\mathrm{P}=0.27)$, LVEDV $(\mathrm{P}=0.15)$, and $\mathrm{CO}(\mathrm{P}=0.17)$

Table 1 Cardiac function data

\begin{tabular}{lccc}
\hline & $\begin{array}{c}\text { Surgical } \\
\text { occlusion }\end{array}$ & $\begin{array}{c}\text { Coil } \\
\text { embolization }\end{array}$ & $P$ value \\
\hline Baseline EF (\%) & $47.6 \pm 6.0$ & $47.3 \pm 7.8$ & $P=0.95$ \\
Final EF (\%) & $50.6 \pm 6$ & $55.8 \pm 8.5$ & $P=0.195$ \\
Baseline ESV (mL) & $24.46 \pm 5.12$ & $25.63 \pm 3.85$ & $P=0.623$ \\
Final ESV (mL) & $32.26 \pm 3.11$ & $28.68 \pm 6.67$ & $P=0.217$ \\
Baseline EDV (mL) & $54.88 \pm 10$ & $48.88 \pm 5.41$ & $P=0.164$ \\
Final EDV (mL) & $65.59 \pm 5.28$ & $64.82 \pm 8.6$ & $P=0.84$ \\
Baseline CO (L/min) & $2.62 \pm 0.66$ & $1.94 \pm 0.32$ & $P=0.021$ \\
Final CO (L/min) & $3.33 \pm 0.56$ & $3.27 \pm 0.81$ & $P=0.885$ \\
\hline
\end{tabular}

Cardiac MRI data at baseline and 35 days post-MI (final) of pigs after surgical occlusion and coil embolization. Values represent the mean $\pm \mathrm{SD}$. EF indicates ejection fraction; ESV, end systolic volume; EDV, end diastolic volume; and CO, cardiac output.
(Figure 2A-D). Two animals from each studied group were represented in Figure 2(E-H), which shows LV wall narrowing (E-F) and myocardial scar after gadolinium contrast injection $(\mathrm{G}-\mathrm{H})$.

\section{Histological and fibrotic changes}

Both Gallego's (Figure 3A) and Masson's (Figure 3B) trichrome staining demonstrated that the remote, border, and infarct zones of all animals were similar in the levels of fibrosis and necrotic tissue. Furthermore, there was no evidence of spontaneous regeneration at sacrifice in either group. Picrosirius red staining was used to analyse the fibrosis scar and confirmed there were no differences between the surgical occlusion and coil embolization groups in CVF (remote zone: $1.77 \pm 0.78$ vs. $1.83 \pm 0.81 \%$, respectively; $\mathrm{P}=0.89$ ) (infarct zone: $51.56 \pm 14.74$ vs. $37.31 \pm 6 \%$, respectively; $\mathrm{P}=0.07$ ), collagen I (remote zone: $0.78 \pm 0.55$ vs. $0.68 \pm 0.34 \%$, respectively; $\mathrm{P}=0.68$ ) (infarct zone: $33.35 \pm 17.04$ vs. $24.36 \pm 13.35 \%$, respectively; $\mathrm{P}=$ 0.35 ), collagen III (remote zone: $1 \pm 0.39$ vs. $1.15 \pm 0.65 \%$, respectively; $\mathrm{P}=0.63$ ) (infarct zone: $18.21 \pm 5.94$ vs. $12.94 \pm$ $13.83 \%$, respectively; $\mathrm{P}=0.38$ ), and the I/III ratio (remote zone: $0.80 \pm 0.52$ vs. $0.81 \pm 0.68 \%$, respectively; $\mathrm{P}=0.97$ ) (infarct zone: $2.35 \pm 2.40$ vs. $15.14 \pm 21.57 \%$, respectively; $\mathrm{P}=0.26$ ) (Figure $3 \mathrm{C}$ ).

\section{Inflammation}

In contrast, inflammation analysis showed significant differences between the surgical occlusion and coil embolization procedures (Figure 4A) when CD3 (3.04 \pm 3.59 vs. $0.35 \pm 0.65$ lymphocytes per field, respectively; $\mathrm{P}=$ $0.002)$ and the $C D 3 / C D 25$ ratio $(10.61 \pm 27.9$ vs. $83.33 \pm$ $40.82 \%$ of activated lymphocytes, respectively; $\mathrm{P}<0.001$ ) were measured in the remote zone and the $\mathrm{CD} 3 / \mathrm{CD} 25$ ratio (33.37 \pm 38.82 vs. $59.94 \pm 35.36 \%$ of activated lymphocytes, respectively; $\mathrm{P}=0.021$ ) was measured in the infarct zone (Figure 4B). Moreover, when remote and infarct zones were compared in each group, there were differences in CD3 $(3.04 \pm 3.59$ vs. $10 \pm 12.33$ lymphocytes per field, respectively; $\mathrm{P}=0.008)$, $\mathrm{CD} 25(0.13 \pm 0.34$ vs. $3.07 \pm$ 3.95 activated lymphocytes per field, respectively; $\mathrm{P}=$ $0.001)$, and the CD3/CD25 ratio (10.61 \pm 27.9 vs. $35.37 \pm$ $38.82 \%$ of activated lymphocytes, respectively; $\mathrm{P}=0.023$ ) in the surgical occlusion group and in CD3 $(0.35 \pm 0.65$ vs. $7.96 \pm 7.24$ lymphocytes per field, respectively; $\mathrm{P}<0.001$ ) and CD25 (0.30 \pm 0.63 vs. $3.82 \pm 3.95$ activated lymphocytes per field, respectively; $\mathrm{P}<0.001)$ in the coil embolization group (Figure $4 \mathrm{C}$ ). In the coil embolization group, the ratio did not differ between the infarct and remote zones, which showed similar activation of the inflammation process throughout the heart $(\mathrm{P}=0.78)$ whereas the activity of lymphocytes was increased in the infarcted myocardium in the surgical occlusion group. 


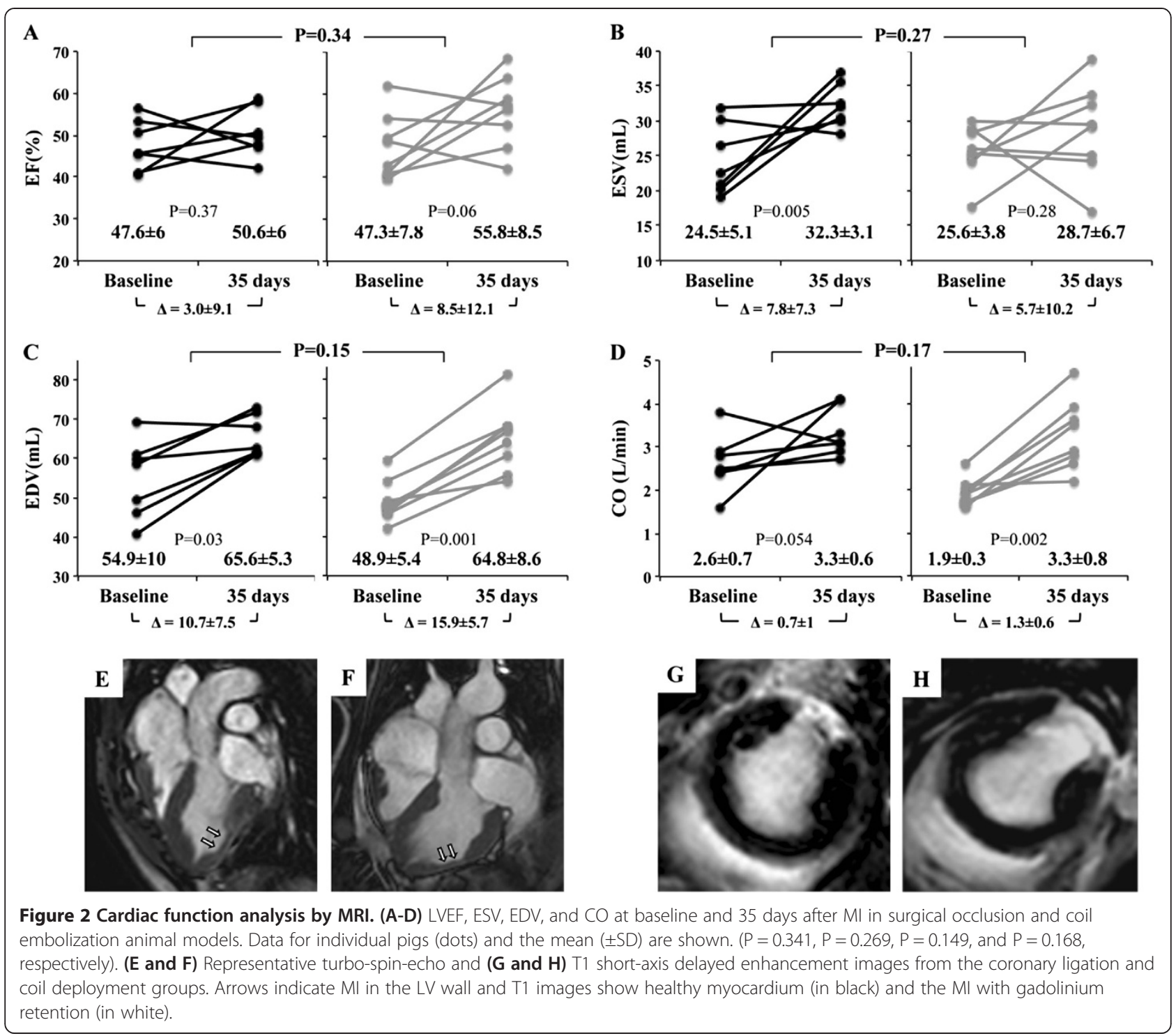

\section{Vascularisation}

After Isolectin B4 staining, no differences in the vascularized area between the surgical ligation and coil deployment groups in remote $(4.08 \pm 2.44$ vs. $3.02 \pm 0.75 \%$, respectively; $\mathrm{P}=0.35)$, infarct $(3.73 \pm 2.79$ vs. $1.74 \pm$ $0.35 \%$, respectively; $\mathrm{P}=0.14)$, and border $(6.76 \pm 9.62$ vs. $3.14 \pm 1 \%$, respectively; $\mathrm{P}=0.4$ ) zones were detected. Nevertheless, coil deployment animals showed significant differences in the vascularized areas when comparing the infarct zone with remote $(\mathrm{P}=0.014)$ and border $(\mathrm{P}=0.004)$ myocardium. All zones appeared similar in the surgical ligation group animals $(\mathrm{P}=0.99 ; \mathrm{P}=0.66$; respectively) (Figure 5 and Additional file 1: Figure S1).

\section{Discussion}

The results of this study confirm that MI, either by surgical occlusion or coil deployment, is similar in terms of infarct size, cardiac function impairment, and myocardial fibrosis, but presents significant differences in myocardial vascularization and inflammation properties when the two techniques are compared.

Currently, MI experimental studies are crucial for testing efficiency of cardiac cell therapy and tissue engineering and ultimately for translational medicine. In this field of research, swine are extremely well suited animals. Several MI models have been suggested for surgical approaches and percutaneous techniques. Although surgical models allow direct visual MI assessment, are feasible, and presume to be easily reproducible, they have an undesired mortality rate (10-50\%) and involve open-chest surgery [12]. Moreover, an open-chest approach increases the risk of infections [13] and comprises postoperative reactive inflammation as well as the establishment of new epicardial collateral vessels [14]. 


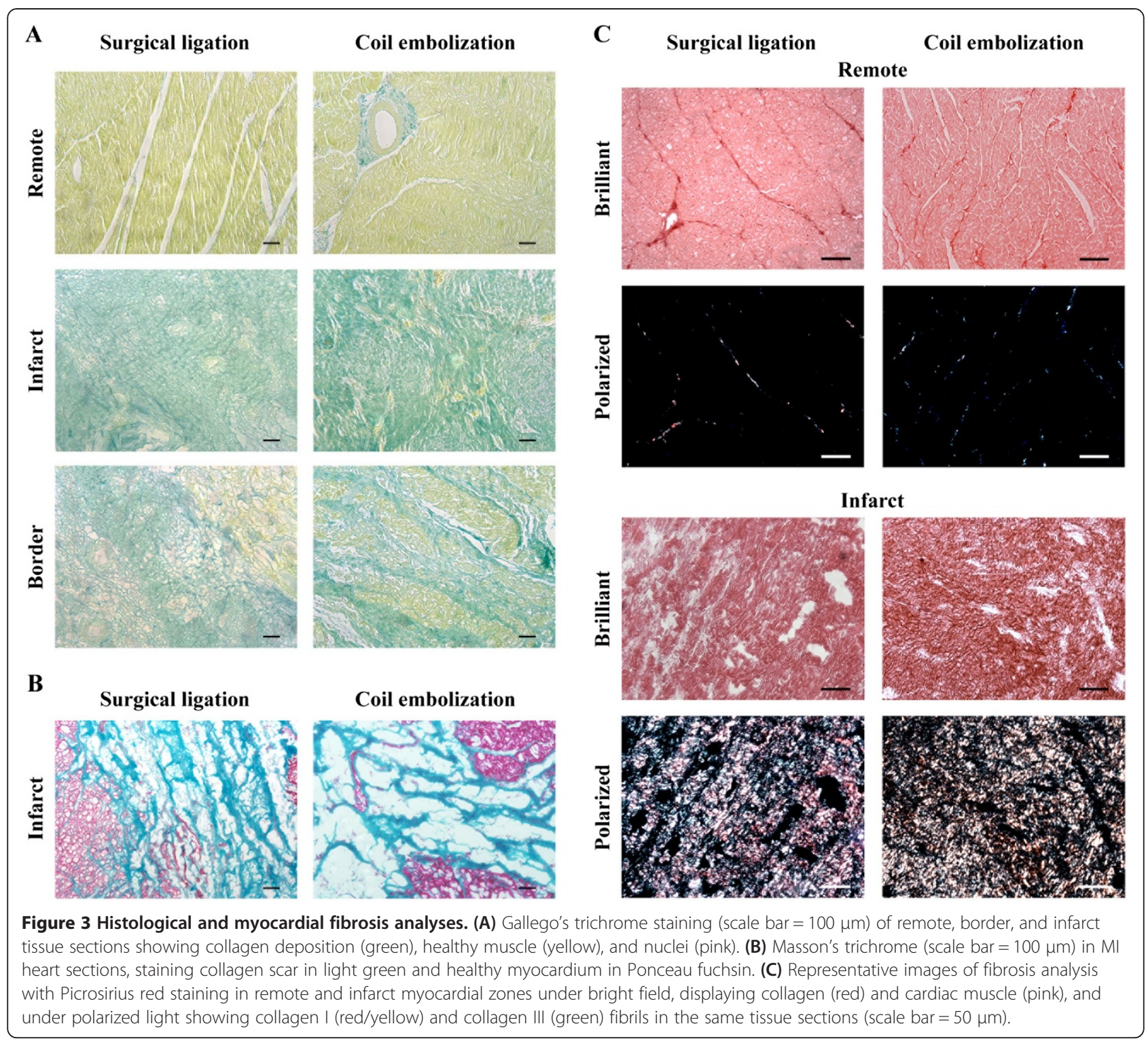

Collectively, these represent substantial differences in comparison with human MI. In the present work a coronary occlusion on the marginal branch of the left circumflex artery was performed to reduce mortality caused by MI induction and evolution. Indeed, in our hands, there was no evidence of arrhythmias or lethal events recorded in these animal series.

Cardiac MRI has become the gold-standard for assessing ventricular function due to its high reproducibility; in addition, this procedure requires fewer animals to evaluate a hypothesis regarding adverse remodeling data [15]. In the present study, MRI analysis demonstrated that global cardiac functional parameters such as LVEF, LVESV, LVEDV and CO did not differ between the two groups 35 days after MI induction. However, LVEF and cardiac output have appeared higher after the MI than at baseline. This result may be partly explained by the juvenile swine model which is still growing with immature cardiac function; by the limited infarct size caused by occluding the first marginal branch of the circumflex coronary artery; and also eventually by cardiac remodelling and neurohormonal activation driven by the cardiac injury. Although there was no LVEF impairment, all animals suffered an adverse remodeling process after MI induction where ventricular volumes significantly increased at sacrifice. It is well known that LVESV is an important parameter to evaluate heart failure progression after $\mathrm{MI}$ and, consequently, is directly responsible for the remodeling phenomena [16,17]. Moreover, myocardial remodeling also includes cell apoptosis, necrosis, and alteration of the balance between myocardial extracellular matrix and collagen fiber synthesis and degradation 


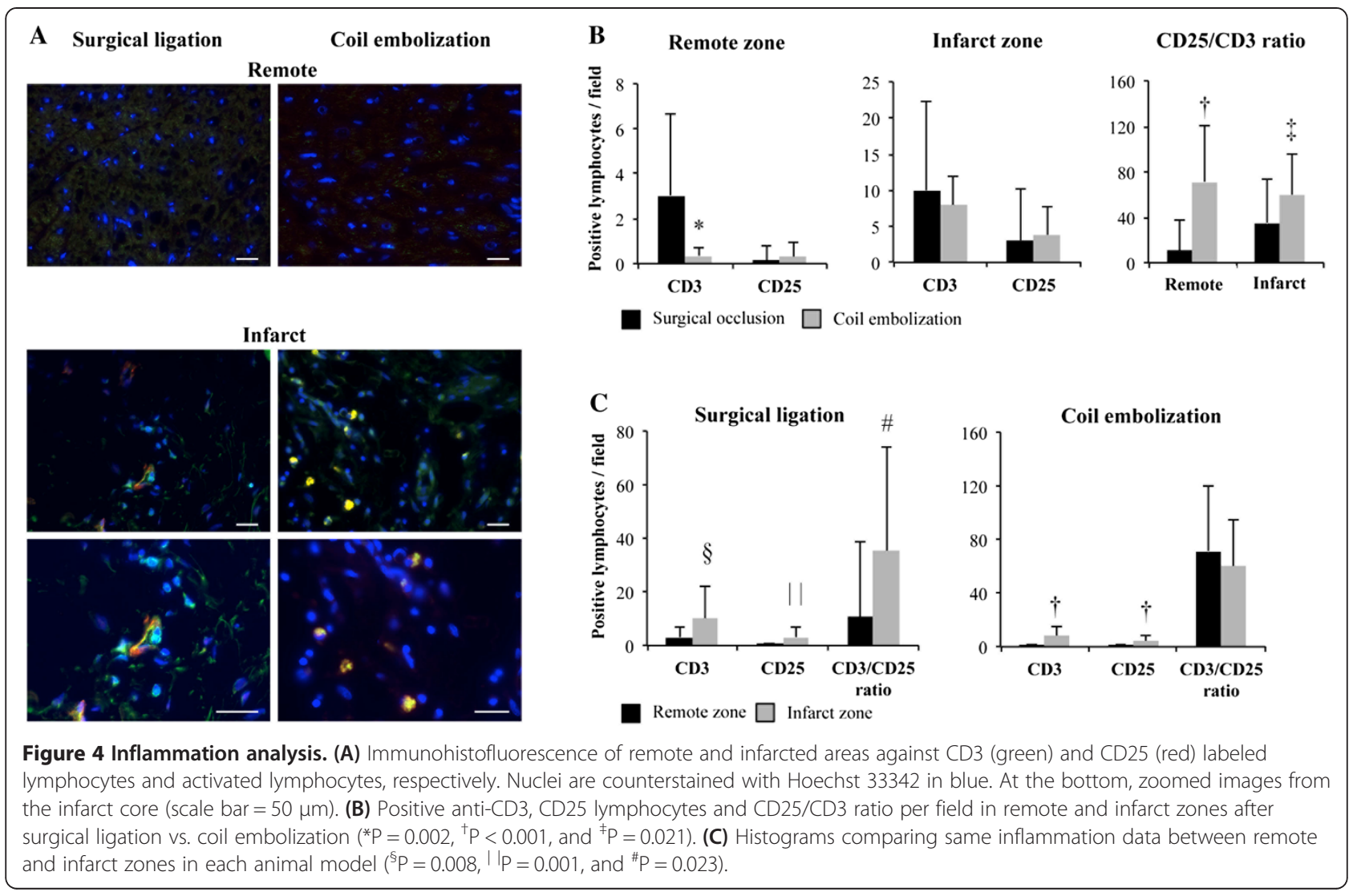

[18]. In this study, myocardial fibrosis was present after MI and was similar in all animals with comparable CVF values and collagen I/III ratios in the infarcted zone.

On the other hand, the inflammatory response after MI is crucial for myocardial healing and repair, diminishing tissue injury and regulating scar formation [19]. The inflammatory process is characterized by an early macrophage and neutrophil infiltration, specific complement activation, secretion of cytokines and, lastly, increasing levels of $\mathrm{T}$ and $\mathrm{B}$ cells [20]. Notably, Varda-
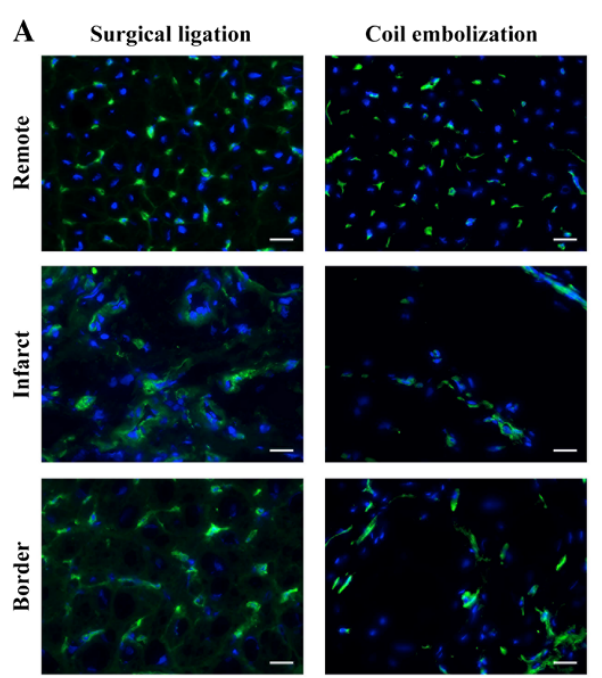

B

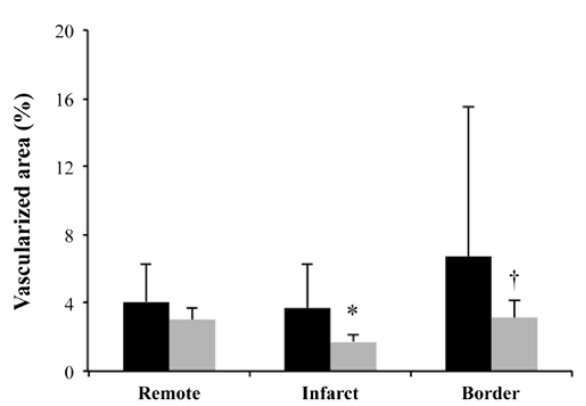

Figure 5 Vascularization analysis. (A) Representative immunohistofluorescence images of Isolectin-B4 (green) and nuclei-Hoechst 33342 (blue) staining showing significant differences in the infarct zone versus remote and border myocardium in the coil embolization model (scale bar $=50 \mu \mathrm{m})$. (B) Percentage of vascularized area in the same myocardial zones ( ${ }^{*} \mathrm{P}=0.014$ and ${ }^{\dagger} \mathrm{P}=0.004$ ). 
Bloom et al. also described a slight mononuclear inflammation in the remote areas of infarcted hearts, finding lymphocytes between healthy muscle fibers. Our findings confirm this presence both in coronary surgical ligation and coil embolization models. In particular, the level of $\mathrm{CD} 3+$ cells in the remote myocardium was notably increased after open-chest model although not so activated as in coil embolization model. In addition, lymphocyte activation (normalized to the total number of $\mathrm{CD}^{+}$ cells) was also increased in the infarcted area of coil animals. Thus, lymphocyte infiltration in the whole heart after MI is more evident after coronary surgical ligation, probably due to the open-chest approach, as Li et al. suggested [13]. However, further long-term studies are needed to evaluate whether lymphocyte activation remains preserved over time leading to deleterious consequences. Furthermore, the higher lymphocyte activation after coil embolization could be explained by the chronic inflammation reaction at the interface between coil and tissue [21]. Hence, the findings presented here support to a better understanding of myocardial inflammation and remodeling processes after MI and also provide new key information to determine the correct MI model.

Interestingly, the growth of subendocardial collateral vessels after acute coronary occlusion has been described both in pigs [22] and humans [23], and was reported as a critical mechanism in the cardiac tissue salvage after injury. As mentioned above, the newly formed neo-vessels after the open-chest approach [11] could also improve myocardial healing, differing from what occurs in the natural human MI process, Although Isolectin B4 staining does not distinguish newly formed vessels, this assumption is suggested from our results due the vascular similarities found between the infarct and border zones with remote healthy myocardium after surgical coronary ligation. On the contrary, myocardial vascularization after MI was significantly diminished in infarcted and border zones in animals operated on the closed-chest approach, as in humans. Although these differences do not alter the infarct size or ventricular function, they should be considered when conducting studies of vascularization after MI. Consequently, coronary surgical occlusion may not be the most representative of clinical coronary ischemic events and alternatives closely resembling pathophysiological traits in humans should be used. Closed-chest percutaneous techniques, which avoid the new collateral development that occurs with open-chest procedures, appear to be a good option, including permanent balloon occlusion and embolization coils [24]. Balloon coronary angioplasty triggers frequent ventricular fibrillation with high mortality rates and its use is better suited in ischemia/reperfusion rather than permanent occlusion studies [12]. In contrast, coil embolization can be applied at any point of the coronary tree to model acute and chronic MI, or chronic congestive heart failure [18]. This alternative appears to be an excellent choice for cell-based therapy, cardiac tissue engineering, gene therapy, and pharmacologic studies in MI in comparison to open-chest coronary occlusion that could interfere with results and may hamper translation to humans.

\section{Conclusion}

In summary, our results support that surgical occlusion and coil embolization MI models generate similar infarct size, cardiac function impairment, and myocardial fibrosis in swine. However, both inflammation and myocardial vascularization levels were closer to those found in humans when coil embolization was performed. Therefore we suggest the use of coil embolization in pre-clinical MI research, being a better MI model than surgical coronary ligation, to obtain reliable results and leading to prompt clinical applications.

\section{Limitations of the study}

Landrace $\times$ Large White prepuberal pigs were used and, thus, results may differ from an adult model. For this purpose, minipigs represent a more regular alternative although they require more complex and expensive handling. Moreover, the ischemic model used in this study produces a limited infarct size, without the induction of malignant ventricular arrhythmias and showing limited effects on ventricular function. In addition, the lack of echocardiographic monitoring prevents the evaluation of ischemic mitral valve regurgitation, frequently observed in lateral LV infarction.

\section{Additional file}

\section{Additional file 1: Figure S1. Vascularization analysis by Gallego's trichrome staining. Representative images showing vascular structures (arrows) in myocardial sections including infarct, border and remote zones after surgical occlusion and coil embolization corroborating Isolectin B4 staining data. Note that Gallego's trichrome technique clearly distinguishes blood vessels (yellowish green) from collagen (dark blue), muscle fibers (yellow), and nuclei (pink). (Scale bar = $100 \mu \mathrm{m}$ ).}

\section{Abbreviations}

MI: Myocardial infarction; ECG: Electrocardiogram; MRI: Magnetic resonance imaging; LV: Left ventricular; LVEF: Left ventricular ejection fraction; LVESV: Left ventricular end systolic volume; LVEDV: Left ventricular end diastolic volume; CO: Cardiac output; CVF: Collagen volume fraction.

\section{Competing interests}

The authors declare that they have no competing interests.

\section{Authors' contributions}

CGM carried out the animal experimental set, participated in its design, performed the statistical analysis, and drafted the manuscript. CPV and IDG helped to carry out the experimental procedures. VC carried out MRI analysis. CSB, SR, ALV and IPG carried out the histological and immunohistochemical analysis and helped in acquisition data. FSM and ABG participated in its design and drafted the manuscript. All authors read and approved the final manuscript. 


\section{Acknowledgements}

The authors want to thank J. Maestre and S. Javato for technical assistance in MRI analysis.

This work was supported by grants from Ministerio de Ciencia e Innovación (SAF2011-30067-C02-01), Fundació La MARATÓ de TV3 (122232), Red de Terapia Celular - TerCel (RD12/0019/0029), and Red de Investigación Cardiovascular - RIC (RD RD12/0042/0027 \& RD12/0042/0047). We also acknowledge the support received from Fundació Privada Daniel Bravo Andreu.

\section{Author details}

'ICREC (Heart Failure and Cardiac Regeneration) Research Program, IGTP, Cardiology Service, Hospital Universitari Germans Trias i Pujol, Crta. Can Ruti, Camí de les Escoles, s/n, 08916 Badalona, Barcelona, Spain. ²Jesús Usón Minimally Invasive Surgery Centre, JUMISC, Cáceres, Spain. ${ }^{3}$ Cardiology Service, Hospital Universitari Germans Trias i Pujol, Badalona, Spain.

${ }^{4}$ Department of Medicine, UAB, Barcelona, Spain.

Received: 19 December 2013 Accepted: 13 May 2014

Published: 21 May 2014

\section{References}

1. Roger VL, Go AS, Lloyd-Jones DM, Adams RJ, Berry JD, Brown TM, Carnethon MR, Dai S, de Simone G, Ford ES, Fox CS, Fullerton HJ, Gillespie C, Greenlund KJ, Hailpern SM, Heit JA, Ho PM, Howard VJ, Kissela BM, Kittner SJ, Lackland DT, Lichtman JH, Lisabeth LD, Makuc DM, Marcus GM, Marelli A, Matchar DB, McDermott MM, Meigs JB, Moy CS, et al: Heart disease and stroke statistics-2011 update: a report from the American Heart Association. Circulation 2011, 123:e18-e209.

2. Windecker S, Bax JJ, Myat A, Stone GW, Marber MS: Future treatment strategies in ST-segment elevation myocardial infarction. Lancet 2013, 382:644-657.

3. Gálvez-Montón C, Prat-Vidal C, Roura S, Soler-Botija C, Bayes-Genis A: Cardiac tissue engineering and the bioartificial heart. Rev Esp Cardiol 2013, 66:391-399.

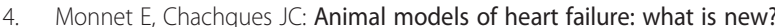
Ann Thorac Surg 2005, 79:1445-1453.

5. Houser SR, Margulies KB, Murphy AM, Spinale FG, Francis GS, Prabhu SD, Rockman HA, Kass DA, Molkentin JD, Sussman MA, Koch WJ, American Heart Association Council on Basic Cardiovascular Sciences, Council on Clinical Cardiology, and Council on Functional Genomics and Translational Biology: Animal models of heart failure: a scientific statement from the American Heart Association. Circ Res 2012, 111:131-150.

6. Liu J, Li X: Novel porcine models of myocardial ischemia/infarctionTechnical progress, modified electrocardiograms validating, and future application. In Advances in Electrocardiograms - Clincal Applications, Volume Chapter 10. Edited by Millis R. Rijeka, Croatia: InTech; 2012:175-190.

7. Kren L, Meluzin J, Pavlovsky Z, Mayer J, Kala P, Groch L, Hornacek I, Rauser P, Vlasin M: Experimental model of myocardial infarction: Histopathology and reperfusion damage revisited. Pathol Res Pract 2010, 206:647-650.

8. Carter-Monroe N, Ladich E, Virmani R, Kolodgie FD: Histopathologic assessment of myocardial regeneration. Methods Mol Biol 2010, 660:125-148.

9. Crisóstomo V, Maestre J, Maynar M, Sun F, Báez-Díaz C, Usón J, SánchezMargallo FM: Development of a Closed Chest Model of Chronic Myocardial Infarction in Swine: Magnetic Resonance Imaging and Pathological Evaluation. ISRN Cardiol 2013, 2013:781762.

10. Gálvez-Montón C, Prat-Vidal C, Roura S, Farré J, Soler-Botija C, Llucià-Valldeperas A, Díaz-Güemes I, Sánchez-Margallo FM, Arís A, Bayes-Genis A: Transposition of a pericardial-derived vascular adipose flap for myocardial salvage after infarct. Cardiovasc Res 2011, 91:659-667.

11. Gálvez-Montón C, Prat-Vidal C, Roura S, Soler-Botija C, Llucià-Valldeperas A, Díaz-Güemes I, Sánchez-Margallo FM, Bayes-Genis A: Post-infarction scar coverage using a pericardial-derived vascular adipose flap. Pre-clinical results. Int J Cardio/ 2013, 166:469-474.

12. Dib N, Diethrich EB, Campbell A, Gahremanpour A, McGarry M, Opie SR: A percutaneous swine model of myocardial infarction. J Pharmacol Toxicol Methods 2006, 53:256-263.

13. Lukács E, Magyari B, Tóth L, Petneházy Ö, Petrási Z, Simor T, Gyöngyösi M, Repa I, Koller A, Róth E, Horváth IG: Evaluation of experimental myocardial infarction models via electromechanical mapping and magnetic resonance imaging. Can J Physiol Pharmacol 2013, 91:617-624.
14. Li RK, Weisel RD, Mickle DA, Jia ZQ, Kim EJ, Sakai T, Tomita S, Schwartz L, Iwanochko M, Husain M, Cusimano RJ, Burns RJ, Yau TM: Autologous porcine heart cell transplantation improved heart function after a myocardial infarction. J Thorac Cardiovasc Surg 2000, 119:62-68.

15. Bellenger NG, Davies LC, Francis JM, Coats AJ, Pennell DJ: Reduction in sample size for studies of remodeling in heart failure by the use of cardiovascular magnetic resonance. J Cardiovasc Magn Reson 2000, 2:271-278.

16. White HD, Norris RM, Brown MA, Brandt PW, Whitlock RM, Wild CJ: Left ventricular end-systolic volume as the major determinant of survival after recovery from myocardial infarction. Circulation 1987, 76:44-51.

17. Migrino RQ, Young JB, Ellis SG, White HD, Lundergan CF, Miller DP, Granger CB, Ross AM, Califf RM, Topol EJ: End-systolic volume index at 90 to 180 minutes into reperfusion therapy for acute myocardial infarction is a strong predictor of early and late mortality. The Global Utilization of Streptokinase and t- PA for Occluded Coronary Arteries (GUSTO)-I Angiographic Investigators. Circulation 1997, 96:116-121.

18. Zhang Y, Shao L, Ma A, Guan G, Wang J, Wang Y, Tian G: Telmisartan delays myocardial fibrosis in rats with hypertensive left ventricular hypertrophy by TGF- $\beta 1 /$ Smad signal pathway. Hypertens Res 2013, doi:10.1038/hr.2013.119.

19. Frangogiannis NG, Smith CW, Entman ML: The inflammatory response in myocardial infarction. Cardiovasc Res 2002, 53:31-47.

20. Varda-Bloom N, Leor J, Ohad DG, Hasin Y, Amar M, Fixler R, Battler A, Eldar $M$, Hasin D: Cytotoxic T lymphocytes are activated following myocardial infarction and can recognize and kill healthy myocytes in vitro. $\mathrm{Mol}$ Cell Cardiol 2000, 32:2141-2149.

21. Böcher-Schwarz HG, Ringel K, Bohl J, Filippi R, Kempski O, Perneczky A: Histological findings in coil-packed experimental aneurysms 3 months after embolization. Neurosurgery 2002, 50:379-384.

22. White FC, Carroll SM, Magnet A, Bloor CM: Coronary collateral development in swine after coronary artery occlusion. Circ Res 1992 71:1490-1500.

23. Cohen MV: Coronary collaterals: clinical and experimental observation. New York, United States: Wiley, John \& Sons, Incorporated; 1985.

24. Peukert D, Laule M, Kaufels N, Schnorr J, Taupitz M, Hamm B, Dewey M: A minimally invasive method for induction of myocardial infarction in an animal model using tungsten spirals. Int I Cardiovasc Imaging 2009, 25:529-535.

doi:10.1186/1479-5876-12-137

Cite this article as: Gálvez-Montón et al:: Comparison of two preclinical myocardial infarct models: coronary coil deployment versus surgical ligation. Journal of Translational Medicine 2014 12:137.

\section{Submit your next manuscript to BioMed Central and take full advantage of:}

- Convenient online submission

- Thorough peer review

- No space constraints or color figure charges

- Immediate publication on acceptance

- Inclusion in PubMed, CAS, Scopus and Google Scholar

- Research which is freely available for redistribution 